\title{
Badara Seck. Il contributo del griot e l'identità come frammento accessorio
}

\author{
Gianpaolo CHIRIACÒ (Bolzano)
}

\section{Summary}

Vocalist, composer, actor and director, Badara Seck was born in Senegal but has been active in Italy for many years. He identifies himself as a griot, and the vast range of his activities can be divided in three areas that are apparently different but can all be related to popular music. Firstly, Seck collaborated with many Italian pop artists, such as Massimo Ranieri and Mauro Pagani. While relying on the visibility earned within the pop scene, Seck articulated a complex strategy in order to be recognized as an authoritative, and "authentic", voice of the migrant communities (second area). Nowadays Badara is mainly focused on leading his own band and releasing original productions (third area).

The three areas are usually considered separately, as it is a hard task - for critics - to fully comprehend that the same Badara whose voice is featured in the soundtrack of the last Checco Zalone's movie is also the author and interpreter of the poignant piece of musical theatre called Galghi. Drawing from Mark Burford's analysis of Sam Cooke's pop albums, I will look at the relationship between Italian popular music and representations of migration in the work of Badara. His work will therefore emerge as a set of "performances [that] conveyed an understanding of ethnicity as an identity that can affirm important solidarities, but also as an accessory to musical performance capable of giving pleasure in any number of ways" (Burford 2012, 141).

Scusate il mio italiano, ma sono colonizzato per la Francia. (Badara Seck)

Vocalist, compositore, attore e regista, Badara Seck è un artista nato in Senegal ma attivo in Italia già da numerosi anni. La sua ampia attività può essere divisa in tre ambiti, apparentemente diversi fra loro ma tutti riconducibili al mondo della popular music. Seck collabora da sempre in veste di special guest con artisti italiani (da Massimo Ranieri a Mauro Pagani). 
Sfruttando la visibilità conquistata in questo modo, segue poi una strategia articolata per farsi riconoscere come voce autorevole (e 'autentica') della comunità migrante. La terza modalità, su cui Badara investe oggi il maggior quantitativo di energie, lo vede leader della sua band e produttore di spettacoli originali.

I tre ambiti vengono solitamente considerati in maniera separata, dal momento che risulta difficile - per i critici - far convivere il Badara che partecipa alla colonna sonora dell'ultimo film di Checco Zalone e un lavoro profondo e articolato come il musical Galghi, di cui Seck è regista e interprete. In questo articolo, seguendo l'esempio di Mark Burford, proverò invece a descrivere la figura del vocalist senegalese osservando tutte le sue anime, nel tentativo di offrire un contributo alla comprensione del complesso rapporto fra popular music italiana e immaginario legato alla migrazione. Il lavoro di Badara Seck emergerà così quale insieme di

performances [that] conveyed an understanding of ethnicity as an identity that can affirm important solidarities, but also as an accessory to musical performance capable of giving pleasure in any number of ways (Burford 2012, 141).

Sebbene faccia ampio uso di metodologie tipiche del lavoro etnografico e antropologico come l'intervista, l'osservazione partecipante, il close playing ${ }^{1}$ - tali metodologie saranno a servizio del tentativo di esplorare i modi in cui la figura e l'opera di Badara Seck possano essere utilizzate come un caso studio, per quanto assai particolare, di come la popular music italiana adotta e allo stesso tempo respinge la figura del migrante, in particolare del migrante nero africano. L'approccio scelto offre un' ulteriore possibilità, quella di presentare il percorso artistico di Badara Seck nello spazio di circa venti anni. L'analisi non si focalizzerà sulla sua posizione in qualità di custode o articolatore di una precisa tradizione musicale, da lui individuata nella parola 'griot' (sui cui significati mi concentrerò). Piuttosto, ciò che si vuole sottolineare in questo contributo sono le modalità attraverso le quali Badara Seck naviga sui confini liquidi dei ruoli (musicali e simbolici) a lui attribuiti, al fine di ri-conquistare continuamente spazi di azione precipui dove la sua musica e la sua visione del mondo possano essere ascoltate attraverso la sua voce.

\section{Il canto e il griot}

Badara Seck è nato a Tivaouane nel 1970. "Appartengo a una famiglia di griot da dieci generazioni. [...] In Africa non esiste la disoccupazione. Se tuo padre è un pescatore, anche tu sei un pescatore. La mia famiglia ha sempre fatto questo mestiere, quello di cantare."2 Griot è un termine diventato popolare, in America prima ancora che in Africa, seguendo un percorso a metà fra connessioni diasporiche e un riduzionismo di comodo (e di consumo) delle culture tradizionali (cf. D'jimo 1989). Come spiega Thomas Hale, il termine 'griot' 
è diventato sinonimo di cantastorie tra la fine degli anni Sessanta e l'inizio degli anni Settanta, grazie anche ad alcune analogie con la figura del bluesman (Oliver 1970); dopodiché il termine è diventato d'uso comune anche in Africa, spesso preferito all'equivalente jali (in Mandinka) o géwél (Wolof) (Hale 1997, 251). La figura del griot nei paesi dell'Africa occidentale era storicamente considerata degna di rispetto: tutori di nobili, diplomatici, consiglieri di regnanti (Hale 1997, 251).

Quando sono arrivate le religioni rivelate - l'islam, il cristianesimo - avevano bisogno della voce dei griot per parlare, per cambiare, per convertire le genti, perché un re, un capo di stato non poteva mai parlare in Africa da solo, sempre c'era un griot che faceva passare il messaggio, che poteva parlare alle genti, cantando con loro o con l'arte di parlare alle genti. Era una forma di arte. ${ }^{3}$

Nei confronti dei griot è prevalso un senso di ambivalenza: rispettati per la loro abilità e libertà d'espressione, sono stati nondimeno vittime di emarginazione sociale, al punto che la pratica di contrarre matrimonio esclusivamente fra i membri delle stesse famiglie di griot risulta ancora oggi molto diffusa. Sebbene orgoglioso delle sue origini, Badara ha molto presto iniziato a viaggiare al di fuori del Senegal. Il desiderio di opporsi a un matrimonio imposto socialmente è stato da subito un importante motore dei suoi viaggi, per quanto tale scelta abbia generato aspre critiche all'interno del suo nucleo famigliare. Un'altra grande motivazione, allo stesso tempo, è stata la sua intenzione di ampliare il bagaglio di esperienze musicali.

Secondo il suo racconto Badara è arrivato in Italia per un caso fortuito. Dopo aver vissuto in Svizzera per alcuni anni, dove ha collaborato con l'arpista svizzero Andreas Vollenweider, Badara Seck aveva partecipato a un tour italiano. Nella tappa milanese di questo tour, qualcosa era andato storto e il cantante si era ritrovato nella difficile condizione di non poter pagare l'albergo per sé e per i suoi musicisti. Il caso ha voluto che in quegli stessi giorni Massimo Ranieri stesse registrando un disco a Milano, nello studio di Mauro Pagani; i due (Ranieri e Pagani) erano alla ricerca di una voce 'etnica' da aggiungere all'arrangiamento. Cosicché, carpita la notizia di un vocalist senegalese in concerto in città, e avendo apprezzato le doti dell'artista senegalese, lo invitarono a registrare con loro e si offrirono per pagare il conto dell'albergo. ${ }^{4}$

Da quel momento in poi, è stato invitato sempre più spesso a lavorare in Italia in collaborazione con vari musicisti. Fino al punto in cui, intorno al $1998^{5}$, Badara decide di spostare la sua residenza a Roma per diventare in breve tempo figura nota nell'ambiente musicale e nella comunità senegalese; nonché volto riconoscibile, talvolta considerato un portavoce, delle comunità migranti nella capitale. 


\section{Il griot e la popular music}

Badara ha continuato a cooperare con Massimo Ranieri e Mauro Pagani per molti anni. Di Ranieri Badara è diventato una spalla fissa nei suoi tour in teatro, almeno fino al 2013-2014. Non ho mai avuto occasione di vedere lo spettacolo dal vivo, ma le informazioni raccolte parlando con chi ha assistito allo show e la documentazione disponibile online ${ }^{6}$ fanno pensare che Badara apparisse soltanto in pochi momenti precisi: intonando alcune melodie in risposta al canto di Ranieri e facendosi coinvolgere in momenti di danze dal sapore swing.

Con Mauro Pagani la collaborazione è stata più saltuaria, ma forse più significativa. Almeno dal punto di vista simbolico, dal momento che Mauro Pagani è considerato una sorta di padre fondatore della world music in Italia, per via del suo ruolo nella concezione e nell'arrangiamento di Crêuza de mä di Fabrizio De André. ${ }^{7}$ Mauro Pagani ha avuto modo di coinvolgere Badara Seck in concerti e produzioni, come per esempio quello della Notte della Taranta del 2007. Uno dei brani che i due realizzano insieme è "Farafrique"8; con lo stesso titolo Badara registra poi un disco nei celebri studi di Pagani. Pubblicato dalla etichetta discografica Officine Meccaniche (sorta di casa discografica collegata agli studi di registrazione), il disco non riceve pareri positivi, e questo contribuisce a creare intorno a lui una fama di musicista "irrispettoso", vittima di una generalizzazione intorno a una non meglio identificata "musica africana” (cf. Pantaleo 2009 e Barachetti 2010). Nonostante le voci critiche, il parere unanime è che la registrazione di Farafrique riveli una voce fuori dal comune. Badara mi ha raccontato in più occasioni di aver registrato un secondo disco negli stessi studi, la cui pubblicazione tuttavia continua a essere rimandata. ${ }^{9}$

In virtù delle sue qualità vocali, Badara Seck è dal 2007 una presenza fissa nei circuiti di world music - lo troviamo per esempio al fianco di Rocco De Rosa - ma allo stesso tempo è diventato una voce richiestissima come special guest ${ }^{10}$ nel campo della popular music. Il modello utilizzato da Ranieri in un brano come "Rundinella", diventa il modello per molte di queste collaborazioni: il leader intona il pezzo sin dall'inizio; la voce di Badara arriva a circa due terzi del suo sviluppo. Nel brano "Se il diluvio scende", registrato da Fiorella Mannoia per Sud, la voce di Badara interviene dopo il secondo ritornello, ma apre una nuova sezione del brano: una coda in cui si alternano le due voci, e che prosegue fino alla fine della traccia. ${ }^{11}$

In tutte queste collaborazioni viene costantemente presentato come 'griot senegalese'. Il termine qui viene inteso nell'accezione 'americana', ovvero come sinonimo di cantastorie, ma sembrerebbe utilizzato per stabilire un'autenticità, come a riconoscere in lui il 'vero' musicista africano. Cosicché la collaborazione fra Badara e grandi nomi del pop - giustificata dal punto di vista musicale da una ricerca di varietà e contrasto timbrico, nonché dalla ricchezza delle trame vocali e dalla eccezionalità del suo timbro - rischia di essere letta come operazione multiculturale di bassa lega, come "multiculturalismo da un punto di vista filantropico: bisogna tollerare la differenza, essere buoni con i bambini neri, aperti con i nostri vicini stranieri, solidali con l'Africa" (Hall/Mellino 2007, 52). E se per alcuni musicisti, come Fiorella Mannoia, collaborare con Badara è parte di una sensibilità al tema della migrazione e di una partecipazione attiva alla discussione politica su questi aspetti, nel caso di 
collaborazioni come quella nella colonna sonora del film di Checco Zalone, il sospetto di voler usare la voce di Badara come segno di apparente rispetto verso una presunta autenticità ( $m a$ all'interno di un'operazione in cui il fine economico soggioga le finalità estetiche) sembra assai solido.

\section{Il griot nelle comunità migranti}

Cristina Lombardi-Diop, studiosa di letteratura e teorica della postcolonialità in Italia, descrive in un articolo il progetto, ormai concluso da tempo, di Roma Residence, un'esperienza di coabitazione costituita da alcuni edifici diventati per qualche anno residenza di individui e comunità appartenenti a diverse provenienze, culture, e religioni. Il ruolo di Badara in Roma Residence è spiegato nel suo articolo:

Badara Seck performs on a regular basis with Italian artist Massimo Ranieri. His visibility outside of Roma Residence represented an asset for those who were inside. When Massimo Ranieri offered Badara an apartment in the prestigious quartiere Flaminio, he refused. As he explained: "I have been living at Residence for three years, I am with my brothers ... I have chosen to stay very close to my brothers in order to offer my help to my brothers. It is normal, it is not only a form of help, it is a complementarity, a way to be close to them ... I live here only for one reason, I tell you, how many times journalists who want to interview me, I call them in at Residence [sic], so that they can come and see... When they arrive, they don't want to enter. This is the main reason why I live here." (Lombardi-Diop 2009, 416-417)

La tattica di Badara si articola a partire da una notevole consapevolezza del proprio ruolo politico e del capitale sociale acquisito grazie all'attività come musicista professionista e alla conseguente visibilità. Nonostante questa tattica possa apparire in contrasto con la figura del cantante sempre pronto a incidere nuove collaborazioni in ambito pop, una lettura di questo tipo terrebbe conto soltanto dell'accezione 'americana' (e poi 'italianizzata') del termine griot. Per Badara rimane invece il riferimento al significato storico del termine: per lui il griot è il consigliere del potere, colui il quale si avvale della propria libertà di parola e si assume la responsabilità di usarla.

Badara è del resto molto chiaro sul suo ruolo in questo senso: concepisce il suo lavoro come quello di un mediatore. Benché per lui si tratti di qualcosa di più di un lavoro, è una sorta di chiamata a impegnarsi per far sì che il dialogo e la consapevolezza reciproca aumentino. "Non esiste il razzismo", afferma spesso durante i concerti, "è solo che non ci conosciamo." Quest'ultima frase è parte di un repertorio di aforismi e aneddoti che Badara utilizza sempre quando parla in pubblico, sul palco per presentare un brano o in risposta a un intervistatore. Un suo aneddoto ricorrente è questo: 
Non è che noi africani non vogliamo lavorare. È che un africano la mattina, prima di andare al lavoro, deve salutare tutto il villaggio. Se ogni saluto dura cinque minuti, ma bisogna salutare tutti, allora la mattina è già finita. Ora, io non dico che salutare tutto il tempo è giusto. L'africano dovrebbe salutare di meno e lavorare di più. Ma voi, qui in Italia, anche voi dovreste lavorare di meno e salutare di più. ${ }^{12}$

Questo esempio ben rappresenta il tipo di comunicazione improntato da Badara, finalizzato alla battuta di spirito ma in grado tuttavia di identificare con esattezza alcuni pregiudizi e di decostruirli. Un altro tipico esempio è la frase usata quasi sempre prima di dire il suo nome sul palco: "Scusate il mio italiano, ma sono colonizzato per la Francia."13

Altro preciso impegno del griot in Italia, a cui tiene molto, è quello di essere una guida per i suoi conterranei in Italia. Più volte l'ho sentito affermare che ricordare ai conterranei i valori della loro terra d'origine è suo compito. Di questo ho avuto una testimonianza diretta durante la presentazione di un libro di poesie del poeta senegalese, da anni residente a Milano, Cheikh Tidiane Gaye. Inserita all'interno di un festival che si tiene ogni anno a Pianopoli, in Calabria, dal titolo Calafrika, la presentazione aveva visto come protagonisti un giovane poeta locale e Cheikh stesso. Arrivato il momento di lasciare spazio al pubblico per eventuali commenti e domande, Badara prende la parola e - microfono alla mano - spiega di non averlo mai incontrato di persona prima di quella sera, ma che tuttavia conosce benissimo la famiglia di Cheikh, giacché la sua famiglia di griot è sempre stata in contatto con quella del poeta. Ma soprattutto - incalza Badara - se l'avesse incontrato a far qualcosa di meno elevato dal punto di vista spirituale, sarebbe stato compito del griot quello di rimproverare il poeta in pubblico. A quel punto, per onorare l'incontro, Badara Seck intona un pekan, presentandolo come un brano della tradizione del Pular, nella regione del Fouta, nel Senegal orientale. Appena intona il brano lo riconosco come una melodia che Badara usa spesso, anche nelle sue collaborazioni - compare ad esempio all'inizio del brano "Farafrique", citato prima - e che sviluppa in maniere diverse secondo il contesto. Durante il canto, Cheikh appare sorpreso dall'inaspettata performance del griot, ma a conclusione dell'intervento ringrazia Badara omaggiandolo con un'offerta di denaro e spiegando al pubblico perplesso che l'offerta in denaro è parte della tradizione: l'orazione del griot si è conclusa pacificamente, senza generare conflitti. O meglio, sciogliendo nelle trame del canto qualsiasi conflitto potenziale.

\section{Badara come leader}

Per tre anni, fra il 2011 e il 2014, non ho mai incontrato Badara. Ma quando l'ho rivisto, nell'estate del 2014, mi ha raccontato di aver interrotto il suo rapporto con Massimo Ranieri, per concentrare gli sforzi sui suoi progetti solisti. Quello del volersi dedicare alla propria carriera, come momento in cui 'finalmente' si può ribaltare il rapporto con il mainstream e con il mercato, per dar vita a prodotti d'arte frutto di ispirazione pura, è un mito ricorrente nella popular music. Lo si incontra continuamente nelle biografie di artisti, in particolare 
degli artisti neri. Un esempio classico è considerare l'esistenza di un Marvin Gaye pre- e post-"What's Going On". Per quanto in un primo momento l'idea di interpretare le parole di Badara in quella chiave mi abbia attratto, riconosco ora che quel tipo di lettura era soltanto il riflesso di un'ideologia - di un mito, appunto - e, come spiegherò più avanti, non ha riscontri nella realtà.

Tuttavia, è vero che il lavoro di Badara dal 2014 in poi riesce a riprodurre più fedelmente il suo punto di vista su temi che nel frattempo sono diventati particolarmente sensibili all'interno del discorso pubblico. Uno su tutti: la migrazione. È del 2014, infatti, un lavoro di teatro musicale, composto e diretto da Badara, dal titolo Galghi. Per questo lavoro, presentato per la prima volta a Roma il 25 giugno di quell'anno, il vocalist senegalese crea un relitto simbolico ('galghi', afferma l'artista, significa 'barca', in wolof), imbastendo il palco come fosse lo scheletro di una qualsiasi delle carrette del mare. Su esso si muovono, parlano, si confrontano e scontrano, cantano, danzano, si coalizzano per affrontare il capitano della nave, uomini e donne diretti a Lampedusa e provenienti da Siria, Palestina, Sudan, Senegal, Camerun. Alcuni degli attori hanno vissuto in prima persona quel viaggio. Ma sono le storie dei protagonisti di Galghi - pur personali - a essere rappresentative delle possibili scelte e dei possibili percorsi di chi attraversa il Mediterraneo.

Nonostante il suo forte impatto visivo e musicale, Galghi ha avuto pochissime repliche. In un precedente lavoro (Chiriacò 2018), ho sostenuto che a causa di un superficiale multiculturalismo operatori e istituzioni culturali non sono stati in grado di comprendere appieno il valore di questo lavoro e la sua unicità. In altre parole, non era stata colta la portata innovativa di una storia contemporanea raccontata da un griot, perché l'idea di un griot come cantore di storie 'tradizionali' era ancora troppo radicata.

Nondimeno, lo spirito di Galghi è ancora molto vivo, e Badara viene spesso invitato a realizzare workshop o incontri all'interno di strutture di accoglienza per ospiti (spesso rifugiati o richiedenti asilo), con i quali realizza nuove collaborazioni, come il brano composto per l'occasione a Bressanone, frutto dell'incontro fra "Tadjaboon", pezzo del repertorio di Badara, e lo spoken word di Karim Doumoya, un richiedente asilo della Costa d'Avorio residente in Alto Adige che ha dato vita a un appassionante progetto di radio via whatsapp dal nome Radio Africani a Bolzano. ${ }^{14}$ Ora è lui, proseguendo con lo stesso spirito collaborativo, a fornire con la sua band uno spazio in cui migranti da poco giunti in Europa possano far sentire la propria voce.

\section{L'identità come frammento accessorio}

Da etnomusicologo interessato alle dinamiche interne alla popular music, ho presentato Badara Seck come un musicista che si identifica come un griot e ho cercato di mostrare come la sua attività si possa dividere in tre sezioni, in ognuna delle quali è all'opera una modalità diversa di assumere e percepire il ruolo del cantore tradizionale. Tuttavia, la mia lettura non è imparziale, nel senso che è evidente una mia predilezione per alcune di queste modalità 
e una mia avversione nei confronti di altre. Non ho resistito alla tentazione di analizzare quella di griot come l'identità principale di Badara Seck, lasciando così che si riverberasse sebbene in una prospettiva critica - l'idea che da uno come Badara ci si aspetti qualcosa di preciso per il solo fatto che si tratti di un musicista nero e senegalese. ${ }^{15}$ Il richiamo di Mark Burford a considerare l'identità etnica come un frammento accessorio, affinché la nostra attenzione di esperti e critici si rivolga all'ampio spettro di alternative possibili tra cui un musicista sceglie per ragioni che hanno a che fare con "politics, profit, and pleasure" (Burford 2012, 169), trova qui il suo senso completo:

Listening that is attentive to a politics of fragments does, however, enable us to acknowledge how facets of a performance, or of a musical career, can work together in harmonious ensemble while also recognizing the occasional resistance of some parts whether repertory, manner of performance, production, or intertextual references - to the whole. (Burford 2012, 167)

Superare una visione unitaria risulta essere un complicato esercizio critico, ma riconosco qui che la mia difficoltà a conciliare Galghi con Checco Zalone allora si possa dissolvere in un ascolto sensibile alle politiche dei frammenti accessori. Pertanto, che sia una strategia di marketing, un'operazione politica, il riflesso di un'ideologia o di una spiritualità, una visione migrante transculturale o un puro divertimento, come interprete del suo lavoro sono tenuto a considerare i mille volti e le apparenti contraddizioni come parti di un progetto artistico coerente. Per quanto mi possa risultare difficile da comprendere, per Badara dedicare del tempo a salutare tutti è parte di quello che è come artista, come migrante contemporaneo, e come uomo.

\section{Note}

1 Per sottolineare il ruolo primario del suono nello studio delle pratiche musicali, Gabriele Solis propone un'analisi ravvicinata di esso, modellata sull'analisi letteraria denominata close reading. Il suo close playing individuerebbe una partecipazione corporea all'evento sonoro che ne colga gli elementi istante dopo istante, esaltando il ruolo relazionale della componente materica del suono (Solis 2012, 530-554). Cf. anche Chiriacò 2016.

2 Sono le parole di Badara in apertura di un seminario tenuto a Bressanone (Bolzano), 16 settembre 2017.

3 Badara, comunicazione personale, 3 agosto 2014.

4 Badara, comunicazione personale, 12 aprile 2017.

5 La data è riportata in un comunicato stampa di Officine Meccaniche: http://www.officinemeccaniche.biz/discografica/it/ (consultazione 10.02.2018). 
$6 \mathrm{Su}$ YouTube sono disponibili alcuni video in cui intona "Rundinella" e "A rumba de scugnizzi”, cf. https://www.youtube.com/watch?v=K4ROMbrq2QY; https://www.youtube.com/watch? v=8WHD7p6FCZY (consultazione 10.02.2018).

7 Da notare che Mauro Pagani aveva già realizzato anni prima un disco in cui sviluppava alcune idee poi presenti in Crêuza de mä (Pagani 1978). A quel disco avevano partecipato diversi componenti degli Area, i quali - secondo quanto confermato anche da Pagani, nel suo racconto biografico (Pagani 2009) - sono stati un'influenza importante nell'elaborazione delle sue idee sulla musica mediterranea. Come ha ben sintetizzato Goffredo Plastino, nella sua analisi di Crêuza de $m \ddot{a}$, "Pagani took up and deepened an interest in the Mediterranean that was already present in Italian pop music" (Plastino 2003, 272).

$8 \mathrm{Su}$ YouTube si può vedere una versione di questo brano per il Festival della Creatività di Firenze del 2007, https://www.youtube.com/watch?v=4oObhTRUcvQ (consultazione 10.02.2018).

9 Il sito www.discogs.com segnala l'esistenza di un altro disco di Badara Seck. Per la precisione viene segnalata come audiocassetta, senza indicazione d'etichetta, e con codice HK 961017, incisa in Svizzera con il titolo Penc. Tuttavia, non sono riuscito ad ascoltare questa incisione, né a ottenere informazioni più precise.

10 Con quest'espressione si intende un musicista/cantante di una certa fama che collabora a una produzione suonando insieme agli interpreti principali in uno o più brani del disco.

11 Si noti come molte delle collaborazioni nell'ambito della popular music, in cui un cantante o un musicista solista interviene come guest, il punto in cui l'ospite appare è spesso lo stesso: dopo la fine del secondo ritornello. Un esempio può essere uno qualsiasi dei tanti brani italiani in cui compare la voce di Caparezza.

12 Dall'incontro con gli studenti dell'Università di Bari Aldo Moro, 12 aprile 2017.

13 In effetti quella usata da Badara è una lingua di contatto, nel senso definito da Taronna 2016.

$14 \mathrm{La}$ performance del brano interpretato da Badara e Karim è visibile online: https://vimeo. com/256074993 (consultazione 10.02.2018).

15 Parafrasando il Fanon di "ero insieme responsabile del mio corpo, della mia razza, dei miei antenati” (Fanon 1996, 99), avverto il rischio che identificare Badara solo come griot mi porti a esprimere posizioni di questo tipo: 'non sei solo responsabile per la tua musica, ma anche per tutti i musicisti neri, e per quello che gli altri africani si aspettano da voi musicisti neri'.

\section{Bibliografia}

Barachetti, Luca: "Badara Seck, Farafrique". In: Sentire Ascoltare (5 febbraio 2010), https://sentireascoltare.com/recensioni/badara-seck-farafrique/ (consultazione 08.02.2018).

Burford, Mark: "Sam Cooke as Pop Album Artist. A Reinvention in Three Songs". In: Journal of the American Musicological Society 65,1 (2012), 113-178.

Chiriacò, Gianpaolo: "Around the Sound: Paesaggi e prospettive tra etnomusicologia e Studi culturali”. In: Vallorani, Nicoletta (ed.): Introduzione ai Cultural Studies. UK, USA e paesi anglofoni. Roma: Carocci, 2016, 143-159. 
Chiriacò, Gianpaolo: "Sound Recordings and Dignity Takings: Reflections on the Racializations of Migrants”. In: Chicago-Kent Law Review 92,3 (2018), in pubblicazione.

D'jimo, Kouyate: “The Role of the Griot”. In: Goss, Linda / Barnes, Marian E: Talk that Talk. An Anthology of African-American Storytelling. New York: Touchstone, 1989, 179-181.

Fanon, Frantz: Pelle nera maschere bianche. Milano: Marco Tropea Editore, 1996.

Goss, Linda / Barnes, Marian E. (ed.): Talk that Talk. An Anthology of African-American Storytelling. New York: Touchstone, 1989.

Hale, Thomas A.: "From the Griot of Roots to the Roots of Griot: A New Look at the Origins of a Controversial African Term for Bard". In: Oral Tradition 12,2 (1997), 249-278.

Hall, Stuart / Mellino, Miguel: La cultura e il potere. Conversazione sui Cultural Studies. Roma: Meltemi, 2007.

Lombardi-Diop, Cristina: “Roma Residence. Senegal, Italy, and Trans-National Hybrid Spaces”. In: Interventions 11,3 (2009), 416-417.

Oliver, Paul: Savannah Syncopators. African Retentions in the Blues. Londra: Stein and Day, 1970.

Pagani, Mauro: Foto di gruppo con chitarrista. Milano: Rizzoli, 2009.

Pantaleo, Rosario: "Badara Seck, Farafrique". In: Folk Bulletin (2009), http://www.folkbulletin.com/ farafrique-badara-seck-officine-meccaniche-2009-griotsenegal/ (consultazione 08.02.2018).

Plastino, Goffredo: "Inventing Ethnic Music. Fabrizio De André's Crêuza de mä and the Creation of Musica Mediterranea in Italy”. In: Plastino, Goffredo (ed.): Mediterranean Mosaic. Local Music, Pattern of Change, Global Contexts. New York/London: Routledge, 2003, 267-286.

Riccio, Bruno: 'Toubab'e 'Vu Cumprà'. Transnazionalità e rappresentazioni nelle migrazioni senegalesi in Italia. Padova: Cleup, 2007.

Solis, Gabriele: "Thoughts on a Interdiscipline. Music Theory, Analysis, and Social Theory in Ethnomusicology". In: Ethnomusicology 61,3 (2012), 530-554.

Taronna, Annarita: Black English. Pratiche linguistiche transfrontaliere Italia-Usa. Verona: Ombre Corte, 2016.

\section{Discografia}

De André, Fabrizio: Crêuza de mä. Ricordi SMRL 6308, 1984 (LP).

Mannoia, Fiorella: Sud. Oyà/Sony Music 88697997732, 2012 (CD/Dig).

Pagani, Mauro: Mauro Pagani. Ascolto ASC 20093, 1978 (LP).

Seck, Badara: Farafrique. Officine Meccaniche OMM 1, 2009 (CD). 